\title{
El sistema de inspección de trabajo respecto al cumplimiento de las normas y derechos laborales en el Perú: A propósito de una revisión sistemática documental
}

\author{
Elsa Mavila Ramírez Pinedo
}

Elsamavilaramirezp@gmail.com

Tarapoto - Perú

\section{RESUMEN}

En los último años, los trabajos de fiscalización, viene siendo aplicado de diferentes modalidades en el campo laboral, el Gobierno por medio de la promulgación de leyes y construcción de organismos fiscalizadores, ha visto necesario adecuar y utilizar distintas tácticas, destinados a proteger y reclamar el respeto de derechos primordiales de los trabajadores; no obstante, se ha podido evidenciar que no resulta suficiente, pues, pese a existir un marco normativo y tener la creación de entes especializados delegados a la fiscalización, la realidad nos sigue mostrando todo lo opuesto.

Siendo una de las funcionalidades principales del Estado la de adoptar mecanismos firmes de defensa de los derechos laborales, es que surge la creación de la Superintendencia Nacional de Fiscalización Laboral (SUNAFIL), constituyendo un componente determinante e importante dentro de lo que conocemos como sistema de inspección de trabajo en el Perú.

De esta forma, la finalidad primordial del presente artículo de revisión sistemática es examinar y analizar los diverso aportes y conclusiones de antecedentes y fuentes documentales relacionadas al sistema de Inspección de Trabajo respecto al cumplimiento de las normas y derechos laborales.

Teniendo como primordial conclusión que existe la necesidad y el valor de conseguir un bien diseñado, eficiente, efectivo y correcto sistema de Inspección de trabajo, que posibilite, comprender en la más grande porción viable el cumplimiento de las normal laborales.

Palabras clave: SUNAFIL; derechos laborales; sistema de inspección de trabajo; empleador. 


\title{
The labor inspection system regarding compliance with labor standards and rights in Peru: regarding a systematic documentary review
}

\begin{abstract}
In recent years, inspection work has been applied in different ways in the labor field, the Government, through the enactment of laws and construction of inspection bodies, has seen it necessary to adapt and use different tactics, aimed at protecting and claiming respect for the fundamental rights of workers; However, it has been shown that it is not enough, because, despite the existence of a regulatory framework and having the creation of specialized entities delegated to the control, reality continues to show us the opposite. Since one of the main functions of the State is to adopt firm mechanisms for the defense of labor rights, the creation of the National Superintendency of Labor Inspection (SUNAFIL) arises, constituting a determining and important component within what we know as the system of labor inspection in Peru.

Thus, the primary purpose of this systematic review article is to examine and analyze the various contributions and conclusions from antecedents and documentary sources related to the Labor Inspection system regarding compliance with labor standards and rights.

Taking as a primary conclusion that there is the need and the value of achieving a well designed, efficient, effective and correct Labor Inspection system, which makes it possible to understand, in the largest viable portion, compliance with labor standards.
\end{abstract}

Keywords: role of SUNAFIL; Labor rights; Labor Inspection System; Employer

Artículo recibido: 10 Setiembre. 2021

Aceptado para publicación: 15 Octubre. 2021 Correspondencia: elsamavilaramirezp@gmail.com Conflictos de Interés: Ninguna que declarar 


\section{INTRODUCCIÓN}

En el campo mundial, especialmente en América Latina, en una indagación elaborada por la Organización Internacional del Trabajo (2011) respecto de trabajo en la economía informal en cuarenta y 7 (47) territorios de medianos y bajos ingresos, se verifica que, la parte importante de trabajo informal está en quince (15) territorios en Latinoamérica (siendo ciertos de dichos: Argentina, Colombia, Brasil, Guatemala, México, Honduras, Perú, Paraguay y Uruguay), es decir, dentro del grupo de organizaciones no registradas o no constituidas en la economía.

Como tenemos la posibilidad de visualizar, a grado de América Latina los empleadores no respetan y/o incumplen con la normativa gremial; o sea, no dan todas las ventajas laborales a sus trabajadores; y, esto, gracias a varios componentes, así sea para ahorrar precios, eludir impuestos, etcétera.; varias de estas razones, fueron definidas por el creador Sandoval (2014). De esta forma además, a pesar de que, existe un sistema de inspección de trabajo, este mecanismo fracasa; ello, según lo amonesta el creador Saco, et al., (2013).

Una vez que se habla de sistema inspectivo de trabajo, se refiere a un sistema incluido, que está formado por diversos órganos y burócratas públicos; los cuales, son indicados y desarrollan sus funcionalidades acordes al marco normativo legal. Mientras tanto que, una vez que se habla sobre cumplimiento de la normativa sociolaboral, involucra el conjunto de leyes que regulan las ventajas que les corresponden a los trabajadores y deberes del empleador.

En varios territorios hay diferentes órganos que salvaguardan los derechos laborales de los trabajadores; y, por ende, el sistema de inspección de trabajo cambia en todos ellos.

En Latinoamérica, el Sistema Inspectivo de Trabajo busca defender las ventajas laborales de los que trabajan, mediante, la ejecución de una inspección en el interior de trabajo al mando de un inspector; y, en caso de verificarse una vulneración de los derechos laborales, existe la imposición de una multa para el empleador. No obstante, a pesar de que, hay una organización pública que fiscaliza y salvaguarda los derechos del trabajador, las organizaciones siguen incumpliendo; lo que, hace percibir que el sistema inspectivo de trabajo no es un sistema eficaz; ello, de acuerdo con la indagación hecha por el creador Casquina (2019). 
Conforme el creador Mendizábal (2019) la problemática que muestra la inspección de trabajo en casi todas las naciones latinoamericanas, son: la corrupción; existe menoscabo del potencial humano y herramientas para el desarrollo de funcionalidades; así como no se brindan capacitaciones especializadas; existe además inconvenientes en el diseño del sistema inspectivo de trabajo.

En Chile, de acuerdo con el creador Rosado (2014) los inconvenientes asociados a la fiscalización del trabajo son: no hay gremios laborales organizados y adaptados a la inspección del trabajo e inexistencia de derechos colectivos. Por esto, en Chile no hay sindicatos que sean eficaces, lo cual, conlleva a que las organizaciones incumplan con abonar las ventajas laborales. Entonces, el sistema inspectivo de trabajo es un ente ineficiente y poco efectivo.

En dicha línea, el 15 de enero del 2013.por medio de la Ley $\mathrm{N}^{\circ}$ 29981, se crea la Superintendencia Nacional de Fiscalización Laboral (SUNAFIL) como un organismo técnico especializado, que se encuentra adscrito al Ministerio de Trabajo y Promoción del Trabajo (MINTRA), responsable de fomentar, fiscalizar y supervisar el cumplimiento del ordenamiento legal sociolaboral y el de seguridad y salud en el trabajo, asimismo, se encarga de proporcionar asesoría especializad y/o técnica, hacer averiguaciones y plantear la emisión de reglas sobre las materias concernientes.

No obstante, resulta fundamental examinar el sistema de inspección de trabajo respecto al cumplimiento de los derechos laborales, teniendo presente que en los últimos años, se vino aplicando de diversas modalidades en el campo meramente laboral, el Estado por medio de la promulgación de leyes y construcción de entes fiscalizadores, ha creído correcto utilizar distintas tácticas, destinados a proteger y reclamar el respeto de los derechos y garantías primordiales del trabajador, sin embargo se puede evidenciar aún, que no resulta suficiente, pues, pese a existir un marco normativo y organismo especializados delegados a la fiscalización, en la realidad se puede evidenciar la vulneración de derechos laborales fundamentales, que incluso se encuentran relacionados con la salud, vida y dignidad de la persona.

\section{ESTRATEGIAS METODOLÓGICAS O MATERIALES Y MÉTODOS}

En el desarrollo del presente artículo científico se revisión sistemática, se tomó en cuenta el aporte y conclusiones de los siguientes antecedentes y fuentes documentales relacionadas al tema: 


\section{A) Antecedentes}

\section{Internacionales}

- Aguayo (2006) en su investigación titulada "La tutela de los derechos laborales" tuvo por objetivo, analizar la tutela de los derechos laborales en Chile”.

- Martínez, Martínez y Rocha (2011) en el estudio denominado "La eficacia de la Inspección del Trabajo en el departamento de León".

- Vial (2015) en su tesis titulada "Dirección del Trabajo: constitucionalidad y límites de la fiscalización laboral"

- Méndez (2012) en su tesis titulada "Causas que originan el incumplimiento de las obligaciones de la Inspección General de Trabajo y que provocan violación de los derechos del trabajador"

\section{Nacionales}

- Julca (2013) en su tesis "Situación de la inspección del trabajo en el Perú y la percepción que tienen de ella las personas que laboran para el sistema y sus usuarios".

- Espinoza (2013) en su tesis "La infracción administrativa laboral”,

- Requejo (2013) en su tesis titulada "Fortalecimiento del sistema de inspección del trabajo en el Perú".

\section{B) Fuente documentales}

- Michael J.; Piore y Schrank en el artículo "Gestión de la flexibilidad e inspección del trabajo en el mundo latino" publicado en la Revista Internacional del Trabajo.

- Rymanov, Alexander y Bocharova, Irina en su artículo "Cuantificación de la resolución de conflictos laborales" publicado en la Revista Dilemas Contemporáneos: Educación, Política y Valores.

- Pernia Hoyo, Nuvia en su artículo "La Inspección del Trabajo y los Entes Administrativos del Estado Venezolano" publicado en Gaceta Laboral.

- Miguel Ángel saco Alva y Diego Campos Skamperle en la publicación del artículo "En búsqueda de un sistema de fiscalización y supervisión laboral adecuado" en la revista IUS ET VERITAS - PUCP”.

- Toyama Miyagusuku, Jorge y Neyra Salazar, Carole a través de su artículo: "Debido proceso, nulidad e inspecciones laborales: ¿qué criterios están aplicando el Ministerio de Trabajo y el Poder Judicial?" publicado en la revista IUS ET VERITAS - PUCP”. 
- Arbulú Alva, Luis, en su artículo: "La Consideración y Aplicación del principio de primacía de la realidad en el procedimiento de inspección del trabajo" publicado en la revista IUS ET VERITAS - PUCP.

\section{RESULTADOS Y DISCUSIÓN}

Aguayo (2006) en su investigación titulada "La tutela de los derechos laborales" concluyó que en una relación de trabajo plasmada a través de un contrato de trabajo, el trabajador es la parte más débil y dependiente de la interacción, algo parecido a ofrecerse voluntariamente para obtener un incentivo económico (cheque de pago) a fin de proteger su esfera personal y familiar. En esta investigación se decidió examinar la legislación dedicada a la tutela de los derechos primordiales de los trabajadores, así como de las personas que renuncien así mismo, a su libertad e independencia a cambio de una indemnización económica. Surgió la necesidad de implementar una sección sustancial en la normativa nacional, pensando en el bien común, en la necesidad de establecer reglas, sistemas y mecanismos para la custodia de este "trabajador débil y vulnerable", priorizando su reconocimiento en su condición de persona, no solamente en público, sino en la mayoría de su tiempo, que abarca su trabajo.

Martínez, Martínez y Rocha (2011) en su investigación denominada "La eficacia de la Inspección del Trabajo en el departamento de León”, obtuvo como conclusión que la inspección del trabajo en el departamento de León avanza y progresa de manera paulatina, para la obtención del respeto del trabajador y sus derechos laborales; consiguió establecer que la efectividad de la tarea inspectiva no alcanza al 50\% de la cantidad de inspecciones que se hacen anualmente, así del análisis que se hizo en relación a los año 2008, 2009 y 2010, el departamento de Managua obtuvo mejor efectividad en sus procesos en estos años, ello debido a que, del número de sus actuaciones inspectivas realizadas, obtuvieron un porcentaje de cumplimiento de $37.66 \%$.

Vial (2015) con su investigación titulada "Dirección del Trabajo: constitucionalidad y límites de la fiscalización laboral” alcanzó a la conclusión que el Régimen por medio de la Dirección de Trabajo tiene la labor de defender los derechos del trabajador referidos a escoger libremente su trabajo; así como observar el cumplimiento de las reglas que regulan la prestación personal de sus servicios. Por otra parte, la organización objeto de análisis, es una entidad administrativa del Estado, especialmente refleja el servicio público no centralizado, por tanto la mayoría de sus actos tienen que constantemente 
regirse al amparo de la legalidad, señalados en los artículos $7^{\circ}$ y $6^{\circ}$ de la Constitución Política de del Perú, base fundamental y rectora del derecho público, lo cual supone que aquel acto emanado de la organización debería ser reflejar las atribuciones otorgadas por ley y encontrarse encuadrada en la competencia que la misma ley le proporciona.

Méndez (2012) con su tesis titulada "Causas que originan el incumplimiento de las obligaciones de la Inspección General de Trabajo y que provocan violación de los derechos del trabajador", alcanzó la conclusión que actualmente no se está cumpliendo con la meta fundamental del Organismo Universal del Trabajo, el cual consiste en fomentar igualdad y oportunidad para varones y damas en lograr trabajos productivos y decentes, mediante condiciones de equidad, independencia, estabilidad y el debido respeto a la dignidad humana.

Julca (2013) en su estudio "Situación de la inspección del trabajo en el Perú y la percepción que tienen de ella las personas que laboran para el sistema y sus usuarios", tuvo como conclusiones que el rol jurídico del Reglamento de inspección del trabajo en nuestro país, se encuentra regulado apropiadamente reconociendo su importancia con rango legal. Sin embargo, la gran aportación de la Ley $\mathrm{N}^{\circ} 28806$ consiste en que los principios constitucionales fueron incorporados en el método de la tarea de la Inspección, siendo complementado con la reposición de la autoridad central, a fin de expandir la competencia de la Inspección de trabajo, robustecer la estabilidad en el trabajo, la autonomía y libertad en las funciones de los inspectores de trabajo además de concordar con el acuerdo 81 de la Organización Internacional del Trabajo.

Espinoza (2013) en su tesis "La infracción administrativa laboral”, tuvo como primordial conclusión que las acciones contradictorias de la tarea administrativa se deberían en cierta medida a la carencia de la regulación y responsabilidad que la parte general del derecho administrativo sancionador no abarca. Es así que, que la finalidad del marco normativo especial que regula la potestad sancionadora sectorial (penal y laboral) circunscriben su tipificación contenida en los delitos y las que corresponden a sanciones, aplicándose solamente en sus respectivos campos.

Requejo (2013) en su tesis titulada "Fortalecimiento del sistema de inspección del trabajo en el Perú", llego a la conclusión que existe una imperiosa necesidad de que el sistema inspectivo del trabajo realice acuerdos de apoyo interinstitucionales con los otros órganos de estado, para conseguir estrategias y uniones eficaces en temas operativos, como 
además para el trueque de datos e información elemental. De esta manera, se reflejará una exclusiva forma para que las inspecciones de trabajo logren llegar a la mayoría de la población.

Michael J., Piore, y Schrank, Andrew en el artículo "Gestión de la flexibilidad e inspección del trabajo en el mundo latino" diferencia el modelo latino, en el cual los inspectores tienen la posibilidad de amoldar las reglas a las necesidades de las organizaciones, y el planteamiento bastante más inflexible de USA. En el modelo latino las reglas laborales tienen la posibilidad de conciliarse con la flexibilidad económica y los inspectores tienen la posibilidad de transformarse en la fuerza de choque de una campaña en pro del trabajo decente. Para eludir que haya comportamientos arbitrarios, habrá que renovar la cultura interna de la inspección, exponer y sistematizar el razonamiento tácito en el cual se fundan las elecciones de los inspectores y hacer nuevos estudios sobre la interacción en medio de las reglas del trabajo y las prácticas que aplican las organizaciones.

Rymanov, Alexander y Bocharova, Irina en su artículo "Cuantificación de la resolución de conflictos laborales" inspeccionan las contradicciones en el campo de las interrelaciones laborales y las maneras modernas de resolverlas en Rusia. Se presta particular atención a la resolución preventiva como la menos controvertida. El análisis muestra que, para incrementar la eficiencia de la inspección del trabajo en la Federación de Rusia en el entorno de las limitadas fuentes de presupuesto de financiamiento, se necesita agrandar el alcance de las ocupaciones de la Oficina del Trabajo. Además, es aconsejable ajustar las ocupaciones prioritarias de las inspecciones de trabajo, primordialmente en la dirección de la utilización de ocupaciones preventivas. El artículo resume la vivencia universal, las superiores prácticas en las ocupaciones preventivas de las inspecciones de trabajo en diferentes territorios de todo el mundo.

Pernia Hoyo, Nuvia en su artículo "La Inspección del Trabajo y los Entes Administrativos del Estado Venezolano", aporta que para el logro de las metas primordiales de la inspección del trabajo, se estricta la cooperación y el trabajo en grupo de los actores de las colaboraciones de trabajo, aquellas sucesivas relaciones entre el Estado, representado por los burócratas de inspección en el trabajo, con los patronos y trabajadores, construyen en grupo la convivencia pacífica y dentro del cumplimiento del marco legal gremial, la 
satisfacción de las necesidades colectivas, por lo cual constituye una herramienta que coadyuva a la concreción dinamizadora de aquellas inter colaboraciones.

Miguel Ángel saco Alva y Diego Campos Skamperle en la publicación del artículo "En búsqueda de un sistema de fiscalización y supervisión laboral adecuado" realizaron un bosquejo de las nociones en general y propiedades del sistema de inspección de trabajo anterior a la construcción de la Sunafil, además, de la manera en la que se vino implementando. Después, se detallan las primordiales noticias que implica la Ley 29981 (Ley que crea la Sunafil) para lograr examinar desde esto, si el novedoso sistema postulado resulta adecuado para una realidad como la nuestra en la que prima la informalidad. Al final, se esboza varias propuestas que servirán como base y camino para conseguir un sistema de inspección correcto, toda vez que una entidad no puede comprender todo, mucho menos, resolver la generalidad de inconvenientes que existe.

Toyama Miyagusuku, Jorge y Neyra Salazar, Carole a través de su artículo: “Debido proceso, nulidad e inspecciones laborales: ¿qué criterios están aplicando el Ministerio de Trabajo y el Poder Judicial?" tratan de conocer cuáles son los criterios más comunes que viene usando tanto el MTPE como el Poder Judicial al instante de iniciar el debido proceso en un proceso inspectivo laboral, para así, con la difusión de dichos criterios, buscar evadir que se repitan actuaciones lesivas a la presunción de inocencia del empleador y al debido proceso, tomando como fundamento la presunción de certeza de los actos administrativos. El presente estudio se basó especialmente en esas actas de infracción que fueron declaradas nulas por decisiones del MTPE al apreciarse transgresiones dentro del proceso inspectivo, que incurrieron en causales de nulidad.

Arbulú Alva, Luis, por medio de su artículo: "La Consideración y Aplicación del principio de primacía de la realidad en el procedimiento de inspección del trabajo", hace un examen sobre el inicio de primacía de la verdad por medio de la inspección de trabajo.

De esta modalidad, la Primacía de la verdad está limitada a la decisión elemental e inicial de vínculos contractuales de naturaleza gremial, sin embargo, cuya aplicación definitiva corresponde al Poder Judicial. Nada impedirá de hecho que la modernidad que se implementa en las interrelaciones de trabajo, en otros términos, en la ejecución de novedosas ocupaciones con enfoques diferentes, realice padecer una revisión y limitación a los inicios del derecho gremial y ofrecer paso, mas bien, a inicios y pautas diferentes. 
En la Revista Internacional del Trabajo, David Weil nos muestra los inconvenientes recientes respecto a la inspección gremial en todo el mundo. De esta forma, nos apunta las próximas deficiencias: (i) escasez de inspectores que realicen sus funciones y lleven a cabo las leyes; (ii) la dificultad de la tarea debido al incremento del sector informal; (iii) insuficiencia de recursos; (iv) escasa capacitación y formación de inspectores; y, (v) la poca infraestructura y carencia de enriquecimiento de apoyo en las ocupaciones inspectivas.

En nuestra realidad peruana, Mauro Ugaz se atrevió a cuestionar el sistema de inspección de trabajo a partir de 3 escenarios bastante concretos: (i) el primero relacionado con la calidad humana, en base a que ningún sistema de fiscalización podría funcionar si no posee a los individuos adecuadas para realizarlo y mucho menos los inspectores no son suficientes; (ii) cuestiona y reprocha los recursos y la capacidad en infraestructura de la entidad que se ocupa de revisar el cumplimiento estricto de las reglas sociolaborales; y, como último escenario, nos presenta al aspecto técnico, en el sentido de no intromisión política. De esta forma, después de equiparar a primera vista las deficiencias planteadas, tenemos la posibilidad de concluir sin lugar a dudas alguna que el Perú no es el exclusivo territorio con inconvenientes en el campo inspectivo.

\section{CONCLUSIÓN O CONSIDERACIONES FINALES}

La funcionalidad del inspector y las reglas y criterios que rigen su comportamiento se concretan en la práctica y por medio de la práctica. Permanecen incrustados en la cultura del organismo, y se transmiten de una generación a otra por medio de la formación profesional y de la vivencia en el trabajo. Evolucionan de igual modo que lo hace la naturaleza de los miembros corporal, atendiendo a lo que pasa en la economía y la sociedad a la que pertenecen y, además, a las prioridades concretas del propio organismo en temas de selección, formación profesional y política general. En este sentido, cabe pensar que los inspectores son los agentes o militares protagonistas de la campaña en pro del trabajo decente.

No se solucionará el problema general solamente con la existencia de una entidad como la SUNAFIL, que por más acciones y operativos inspectivo que viene realizando a partir de su funcionamiento, necesita de un cambio en la mentalidad de los empleadores que muchas veces prefieren el pago de sanciones, antes que dar cumplimiento a los derechos laborales de los trabajadores; más aún cuando la gran proporción de 
inconvenientes que los trabajadores aquejan en el entorno peruano se debe a la informalidad de los empleadores.

Aun cuando hubo un aumento relevante de las inspecciones de trabajo su predominación en la regulación laboral aún resulta insuficiente, para lograr fiscalizar el cumplimiento de derechos laborales en regiones rurales, organizaciones informales e ilegales, así como micro organizaciones.

No obstante, se aprecia además que, por la monumental carga de trabajo, la pluralidad de actuaciones que se resultan inspecciones complicadas; aunado al poco tiempo para averiguar y recabar medios de prueba; se suma el hecho que hay actuaciones inspectivas que no cuentan con el soporte técnico y legal correcto, tomando como premisa la presunción de certeza de las actuaciones administrativas, de esta manera se está imponiendo sanciones que están afectando la presunción de inocencia del empleado y al debido proceso. Otro problema de la inspección de hoy, es la falta de unificación de criterios normativos aplicables a casos concretos, originado de la intromisión de sistemas políticos en muchas oportunidades pues en algunas zonas los inspectores de trabajo dependen del gobierno Regional y no propiamente del Ministerio de Trabajo. De esta forma, en un Gobierno Regional que designe y configure el proceso de inspección de determinado sujeto inspeccionado, podría hacerse de forma "y", en otra zona se plantea que debería ser de forma " $x$ ", y en Lima de manera " $z$ ", existiendo diversidad de criterios.

A partir de un criterio operativo, no conseguir y alcanzar a aquellos sectores más vulnerables y necesitados que resultan siendo mayormente los sectores informales, continuará siendo una gran traba y reto para la SUNAFIL y el sistema de inspección de trabajo, ello se debe a que los inspectores no llegan a estos centros laborales donde se vulneran derechos fundamentales relacionados incluso con la seguridad y salud en el trabajo. En este punto, debemos recordar, que las inspecciones que se generan de oficio son contadas con los dedos de las manos; pues el inspector de trabajo actúa fundamentalmente por acusación y/o denuncia, lo cual ocurre habitualmente en organizaciones formalizadas. En impacto, el problema no consiste, en esencia, en generar un mecanismo de sanción ante los problemas y diferencias surgidos como resultado de las interrelaciones de trabajo, lo cual muchas veces resulta irreparable. Más bien, creemos que un sistema de fiscalización conveniente debería operar desde un escenario preventivo de probables males y afectaciones al trabajador, que resulta ser la parte más débil de la relación laboral; por ello, 
es importante interiorizar que la fiscalización es la roca angular en evitar y prevenir los riesgos laborales.

Existe la necesidad y el valor de conseguir un bien diseñado, eficiente, efectivo y correcto sistema de Inspección de trabajo, que posibilite, con las lagunas, vacíos y deficiencias que existen en todo sistema, comprender en la más grande porción viable el cumplimiento de las reglas laborales.

Para efectividad del sistema de inspección en el trabajo, los organismos que lo conforman tienen que cooperar entre sí. La inspección en el trabajo debería reinventarse para no perder vigencia, teniendo presente que el objetivo del sistema de inspección de trabajo no es solo sancionar, sino producir una cultura de cumplimiento de obligaciones y respeto de derechos laborales.

\section{LISTA DE REFERENCIAS}

Arbulú Alva, L. (2005) “La Consideración y Aplicación del principio de primacía de la realidad en el procedimiento de inspección del trabajo" Revista IUS ET VERITAS. Recuperado de http://revistas.pucp.edu.pe/index.php/derechoysociedad/article/view/16972/17273.

Julca, M. (2013). Situación de la inspección del trabajo en el Perú y la percepción que tienen de ella las personas que laboran para el sistema y sus usuarios. Universidad Nacional Mayor de San Marcos. Recuperado de: http://cybertesis.unmsm.edu.pe/handle/cybertesis/3473.

Ley Nº 28806. (2006). Ley General de Inspección del Trabajo. Congresso de la República del Perú.

Martínez, B., Martínez, M. y Rocha, J (2011). “La eficacia de la Inspección del Trabajo en el departamento de León”, Recuperado de: http://riul.unanleon.edu.ni:8080/jspui/retrieve/230, el 15 de diciembre de 2017.

OIT (2015). Organización Internacional del trabajo. Inspección Laboral. Recuperado de: http://www.ilo.org/global/standards/subjects-covered-by-international-labourstandards/labour-inspection/lang--es/index.htm

Pernia, N. (2013). "La Inspección del Trabajo y los Entes Administrativos del Estado Venezolano. Publicación de Gaceta Laboral. Vol. 19. Recuperado de (http://web.b.ebscohost.com/ehost/pdfviewer/pdfviewer?vid=24\&sid=3fc00048c192-45d0-8c9b-b4ec25e5bfa2\%40pdc-v-sessmgr04 
Piore, M y Schrank, A.(2008), "Gestión de la flexibilidad e inspección del trabajo en el mundo latino". Revista Internacional del Trabajo, Vol. 127. Recuperado de http://web.b.ebscohost.com/ehost/pdfviewer/pdfviewer?vid=17\&sid=3fc00048c192-45d0-8c9b-b4ec25e5bfa2\%40pdc-v-sessmgr04

Requejo, J. (2013). Fortalecimiento del sistema de inspección del trabajo en el Perú. Pontifícia Universidad Católica del Perú. Recuperado de: http://tesis.pucp.edu.pe/repositorio/bitstream/handle/123456789/5239/REQUEJO_ ALEMAN_JUAN_INSPECCION_TRABAJO.pdf?sequence $=1$.

Rymanov, A y Bocharova, I.(2019). “Cuantificación de la resolución de conflictos laborales” (Spanish). Revista Dilemas Contemporáneos: Educación, Política y Valore. Recuperado de http://web.b.ebscohost.com/ehost/pdfviewer/pdfviewer?vid=22\&sid=3fc00048c192-45d0-8c9b-b4ec25e5bfa2\%40pdc-v-sessmgr04

Saco, M y Campos, D. (2013): "En búsqueda de un sistema de fiscalización y supervisión laboral adecuado: A propósito de la creación de la Superintendencia Nacional de Fiscalización Labora”. Revista IUS ET VERITAS, N 46. Recuperado de http://revistas.pucp.edu.pe/index.php/iusetveritas/article/view/11983/12551

Servat, R. (2001). La inspección del trabajo y la defensa del trabajador. Recuperado de: http://chehadeabogados.com/documentos/documentos/ pdfs/DL_910_Nueva_Ley_de_Inspecciones.pdf

Soriano, M. (2014). Situación de la inspección del trabajo en el Perú y la percepción que tienen de ella las personas que laboran para el sistema y sus usuarios. Recuperado de:

http://chehadeabogados.com/documentos/documentos/pdfs/DL_910_Nueva_Ley_d e_Inspecciones.pdf

Toyama Miyagusuku, J y Neyra Salazar, C (2011) "En búsqueda de un sistema de fiscalización y supervisión laboral adecuado: A propósito de la creación de la Superintendencia Nacional de Fiscalización Labora". Revista IUS ET VERITAS Recuperado de http://revistas.pucp.edu.pe/index.php/iusetveritas/article/view/12088/12655

WEIL, David. Un Planteamiento Estratégico de la Inspección del Trabajo. En: Revista Internacional del Trabajo. Volumen 127. Boston: 2008; p. 390. 